\title{
Biodiversity, extinctions, and evolution of ecosystems with shared resources
}

\author{
Vladimir Kozlov* \\ Department of Mathematics, University of Linkoping, 58183 Linkoping, Sweden \\ Sergey Vakulenko \\ Institute for Mechanical Engineering Problems, Russian Academy of Sciences, Saint Petersburg, Russia \\ and Saint Petersburg National Research University of Information Technologies, Mechanics and Optics, Saint Petersburg, Russia \\ Uno Wennergren \\ Department of Ecology, University of Linkoping, 58183 Linkoping, Sweden
}

(Received 18 January 2017; published 21 March 2017)

\begin{abstract}
We investigate the formation of stable ecological networks where many species share the same resource. We show that such a stable ecosystem naturally occurs as a result of extinctions. We obtain an analytical relation for the number of coexisting species, and we find a relation describing how many species that may become extinct as a result of a sharp environmental change. We introduce a special parameter that is a combination of species traits and resource characteristics used in the model formulation. This parameter describes the pressure on the system to converge, by extinctions. When that stress parameter is large, we obtain that the species traits are concentrated at certain values. This stress parameter is thereby a parameter that determines the level of final biodiversity of the system. Moreover, we show that the dynamics of this limit system can be described by simple differential equations.
\end{abstract}

DOI: 10.1103/PhysRevE.95.032413

\section{INTRODUCTION}

Thermodynamics and statistical physics allow us to describe the equilibrium states of systems consisting of many particles with a few variables. This approach is very effective for many physical and chemical applications, particularly when we are dealing with closed systems. It would be very tempting to find such reduced macroscopic descriptions for large ecological and economic systems.

Recently [1], a very reduced description was proposed for a class of ecological models. It is based on an analog of the meanfield theory, and it aims to describe possible bifurcations. In the present paper, we suggest a variant of a reduced description for another large class of ecosystems, where many species compete for a few resources. Our second goal is to consider one of the most intriguing puzzles in ecosystem theory, namely the biodiversity problem: why can such a large number of similar species share the same habitat, and how do we estimate this number? A typical example is an aquatic ecosystem where many different phytoplankton species coexist. The principle of competitive exclusion $[2,3]$ asserts that different species sharing the same resource cannot coexist, and it predicts that an assembly of competing species will converge to a single species. In general, competition models show that the number of species that can coexist in equilibrium cannot be greater than the number of limiting factors $[4,5]$. However, hundreds of species of phytoplankton coexist despite the fact that nitrate, phosphate, light, and carbon are the only resources regulating phytoplankton growth [6,7]. It is not clear why this is, although the problem has attracted a great deal of attention among ecologists (see, for example, [8-10], among many others), and they have suggested numerous different approaches to

\footnotetext{
*vladimir.kozlov@liu.se
}

that problem based on game theory, chaos, stochastics, space inhomogeneities, turbulence, etc.

In this paper, we consider a model of an ecological system in which many species share the same resource. Our dynamical equations are close to the equations considered in [11], but we extend that model to take into account species extinctions and self-limitation effects (which are important, in particular, for plankton populations [7]). There are a number of works devoted to evolution in a multispecies context such as food webs (see, for example, [12-15]) as well as the effect of species extinctions $[16,17]$. These papers have used the Lotka Volterra model, in which species interactions slowly evolve over time (this may be connected with foraging [16] or an adaptation of species behavior), and species extinctions are possible when the abundances attain a critical threshold. In our model, all of the parameters are random. However, contrary to $[16,17]$, they are fixed in time, and the primary effect on evolution is species extinctions.

The main results are as follows. We introduce a specific numerical characteristic, which we will call the stress parameter $P_{\text {stress. }}$. It is a natural dimensionless multiplicative combination of some main parameters involved in the model formulation, namely the resource turnover rate, the maximum supply of the resource, the self-limitation coefficient, and the averaged specific growth rates. The stress parameter appears, in a natural way, as a result of a model rescaling, and it can be interpreted as a magnitude of selection pressure on ecosystem species induced by the interaction between the ecosystem and its environment. For example, $P_{\text {stress }}$ is large if the resource amount or the resource turnover rate is small. For large values of the stress parameter, the model exhibits an effect of convergence to similarity as in the competitive Lotka-Volterra systems studied earlier [18]. In contrast to [18], we obtain a complete analytical description of the system behavior. We show that the model with or without extinction thresholds 
is sharply different. Namely, the simpler model without a critical extinction threshold exhibits a global stability, which occurs when all positive trajectories converge to the same equilibrium independent of the initial state. The model with extinction dynamics is fundamentally nonpredictable. The trajectories tend to different equilibria, and these final states depend on initial data. The biodiversity level, which we observe after a long evolution, can be expressed via the initial number of species, the stress parameter, and other ecosystem characteristics in an explicit way. This convergence to similarity in a species trait can be called a "concentration effect." However, this concentration effect does not mean that all species are completely identical: other traits defined by species parameters can still differ.

The concentration effect leads to interesting phenomena. Let us suppose that initially an ecosystem contains a number of species with random parameters, and let us consider the limit system, which is a result of a long evolution. We compare how a sharp change in environment can affect the initial and final systems. With our analytical relations one can estimate the number of species that may become extinct. The limit system is more stable than the initial one.

The limit ecosystem, which arises as a result of a long evolution, has interesting properties: its dynamics is governed by a simple differential equation of second order. This equation describes a nonlinear oscillator with a friction and a memory. If the friction is small and the memory is negligible, the dynamics of this oscillator is defined by a Hamiltonian system. The formation of this universal limit system is a result of species extinctions and a selection pressure on some species parameters, namely those that are important for species survival.

\section{POPULATION DYNAMICS}

We consider the following system of equations:

$$
\begin{gathered}
\frac{d x_{i}}{d t}=x_{i}\left[-r_{i}+\phi_{i}(v)-\gamma_{i} x_{i}\right], \quad i=1, \ldots, M, \\
\frac{d v}{d t}=D\left(S_{0}-v\right)-\sum_{i=1}^{M} c_{i} x_{i} \phi_{i}(v),
\end{gathered}
$$

where

$$
\phi_{i}(v)=a_{i} \Phi\left(v, K_{i}\right), \quad \Phi(v, K)=\frac{v}{K_{i}+v} .
$$

Here $x_{i}$ are species abundances, $M$ is the number of species, $v$ is the resource amount, $D$ is the resource turnover rate, and $S_{0}$ is the maximum supply of resource $v$. In total, there are five species-specific parameters: $r_{i}$ are species mortality, $c_{i}>0$ is the fraction of resource consumption by individuals of the $i$ species, $\gamma_{i}>0$ defines species-specific self-limitation, the coefficients $a_{i}>0$ are species-specific growth rates, and $K_{i}>0$ are species-specific resource constants indicating a reduction of resource effect by half. For $\gamma_{i}=0$, this system has been used to study the plankton paradox [11]. Following [7], we assume $\gamma_{i}>0$ since it is known that self-limitation is essential for large ecosystems $[19,20]$ and that plankton and plant ecosystems can induce effects leading to self-limitation [7]. We complement the system (1) and (2) with the initial conditions

$$
x_{i}(0)=\bar{x}_{i}, \quad v(0)=v_{0} .
$$

\section{GLOBAL STABILITY FOR THE MODEL WITHOUT EXTINCTIONS}

Here we show that the Cauchy problem (1), (2), and (4) has a positive solution for all positive Cauchy data. Furthermore, we study both the stability and large time behavior of solutions.

Proposition I. Solution $(x(t), v(t))$ of (1) and (2) with initial data $v(0) \geqslant 0, \bar{x}_{i}=x_{i}(0) \geqslant 0$ is defined for all positive $t$, and it satisfies the estimates

$$
0 \leqslant x_{i}(t) \leqslant \frac{\bar{x}_{i} \exp \left(\bar{a}_{i} t\right)}{1+\bar{x}_{i} \gamma_{i} \bar{a}_{i}^{-1}\left[\exp \left(\bar{a}_{i} t\right)-1\right]},
$$

where $\bar{a}_{i}=a_{i}-r_{i}$, and

$$
0 \leqslant v(t) \leqslant S_{0}[1-\exp (-D t)]+v(0) \exp (-D t) .
$$

Proof. Since $\phi_{i}(v)<a_{i}$, we have $x_{i}(t) \leqslant y_{i}(t)$, where $y_{i}(t)$ is the solution of the Cauchy problem,

$$
\frac{d y_{i}}{d t}=y_{i}\left(-r_{i}+a_{i}-\gamma_{i} y_{i}\right), \quad y_{i}(0)=\bar{x}_{i} .
$$

Solving this equation, we obtain (5). Estimate (6) follows from the non-negativity of the term $\sum_{i=1}^{M} c_{i} x_{i} \phi_{i}(v)$.

\section{A. Global stability}

Let

$$
X_{i}(v)=\left(\Phi\left(v, K_{i}\right)-p_{i}\right)_{+}, \quad p_{i}=r_{i} / a_{i},
$$

where $z_{+}=\max \{z, 0\}$, and let also

$$
F_{M}(v)=F_{M}(v, b, K, p)=\sum_{i=1}^{M} R_{i}(v, b, K, p),
$$

where

$$
R_{i}(v, b, K, p)=b_{i} \Phi\left(v, K_{i}\right) X_{i}(v) e, \quad b_{i}=c_{i} \gamma_{i}^{-1} a_{i}^{2} .
$$

Here the quantity $R_{i}$ can be interpreted as a consuming rate of the $i$ th species, and $F_{M}$ is the sum of all consuming rates.

Here $a=\left(a_{1}, \ldots, a_{M}\right), b=\left(b_{1}, \ldots, b_{M}\right), p=\left(p_{1}, \ldots, p_{M}\right)$, and $K=\left(K_{1}, \ldots, K_{M}\right)$. If $v$ is a non-negative root of the equation

$$
D\left(S_{0}-v\right)=F_{M}(v, b, K, p)
$$

then

$$
x_{i}=a_{i} \gamma_{i}^{-1} X_{i}(v), \quad i=1, \ldots, M,
$$

and $v$ is an equilibrium point of the system (1) and (2). We assume here and in what follows that

$$
\max _{i}\left(\Phi\left(S_{0}, K_{i}\right)-p_{i}\right)>0 .
$$

Then the function $F_{M}$ is non-negative for $v \geqslant 0, F_{M}(v)=0$, and $F_{M}\left(S_{0}\right)>0$ due to (11). This implies that Eq. (9) has a unique non-negative solution, which belongs to the interval $\left(0, S_{0}\right)$. We denote this solution by $v_{\text {eq }}$. 
Let us rewrite Eq. (9) in the following way. Consider first the relation

$$
v=S_{0}-\frac{1}{D} \sum_{i=1}^{M} b_{i} \Phi\left(v, K_{i}\right) X_{i}(w)=G(v, w),
$$

with $w \in\left[0, S_{0}\right]$. Since $G(v, w)$ is decreasing in $w$ from $S_{0}$ to something that is smaller than $S_{0}$, for each $w$ the above equation has a unique solution $v=V(w)$. One can verify that the function $V$ is nondecreasing and continuous, $V(0)=S_{0}$ and $V\left(S_{0}\right)>0$. We can consider (9) as the following fixedpoint equation:

$$
v=V(v), \quad v \in\left[0, S_{0}\right] .
$$

To describe the large time behavior of the system (1) and (2), we consider the following iterative procedure of solving (12):

$$
v^{(k+1)}=V\left(v^{(k)}\right), \quad k=0,1, \ldots, \quad \text { and } \quad v^{(0)}=0 .
$$

Then $v^{(1)}=S_{0}$, and $v_{2}$ is the solution to

$$
v^{(2)}=G\left(v^{(2)}, S_{0}\right),
$$

which is positive due to assumption (11). Since $V$ is nondecreasing, we have

$$
0=v^{(0)}<v^{(2)} \leqslant v^{(4)} \leqslant \cdots \quad \cdots \leqslant v^{(3)} \leqslant v^{(1)}=S_{0} .
$$

We set

$$
\hat{v}=\lim _{k \rightarrow \infty} v^{(2 k)} \quad \text { and } \check{v}=\lim _{k \rightarrow \infty} v^{(2 k+1)}
$$

Clearly,

$$
0<v^{(2)} \leqslant \hat{v} \leqslant v_{\text {eq }} \leqslant \check{v} \leqslant S_{0} .
$$

Moreover,

$$
\hat{v}=S_{0}-\frac{1}{D} \sum_{i=1}^{M} b_{i} \Phi\left(\hat{v}, K_{i}\right) X_{i}(\check{v})
$$

and the same relation holds if $\hat{v}$ and $\breve{v}$ are exchanged. Now we can formulate our main result about the large time behavior of solutions to (1) and (2).

Theorem I. Let $(x(t), v(t))$ be a solution of (1) and (2) with positive initial data. Then

$$
\liminf _{t \rightarrow \infty} v(t) \geqslant \check{v}, \quad \limsup _{t \rightarrow \infty} v(t) \leqslant \hat{v}
$$

and

$$
\liminf _{t \rightarrow \infty} x_{i}(t) \geqslant X_{i}(\check{v}), \quad \limsup _{t \rightarrow \infty} x_{i}(t) \leqslant X_{i}(\hat{v}),
$$

$i=1, \ldots, M$.

For the proof of this theorem, see the Appendix.

Note that $\hat{v}=\check{v}$ if $d V / d w>-1$, which is true when, for example, $D$ or $\gamma_{0}=\min _{i} \gamma_{i}$ are sufficiently large. Indeed, if $d V / d w \in(-1,0]$, the operator $v \rightarrow V(v)$ defined on $\left[0, S_{0}\right]$ is a contraction and therefore the iterations $v^{(k)}$ converge to the same limit. This observation implies the following:

Corollary $I$. For sufficiently large $D>0$ or $\gamma_{0}>0$, all the solutions $(x(t), v(t))$ of (1) and (2) with positive initial data converge, as $t \rightarrow \infty$, to the unique equilibrium point defined by Eqs. (9) and (10).

\section{B. Local stability}

Consider now the problem of stability of equilibrium states $\left(x_{1}, \ldots, x_{M}, v_{\mathrm{eq}}\right)$ defined by (9) and (10). Denote by $\mathbf{I}_{\mathrm{eq}}$ the set of indices $i$ for which $\phi_{i}\left(v_{\mathrm{eq}}\right)-r_{i}>0$ and by $N_{\mathrm{eq}}$ the number of such indices. Then $x_{i}>0$ when $i \in \mathbf{I}_{\text {eq }}$.

One can show that the eigenvalues of the linear approximation of (1) and (2) at the equilibrium point $\left(x_{1}, \ldots, x_{M}, v_{\text {eq }}\right)$ satisfy the equation (see the Appendix)

$$
\lambda+D+G(\lambda)=0,
$$

where

$$
G(\lambda)=\sum_{i \in \mathbf{I}_{\mathrm{eq}}} c_{i}\left(x_{i} \phi_{i}^{\prime}\left(v_{\mathrm{eq}}\right)+\phi_{i}\left(v_{\mathrm{eq}}\right) \frac{x_{i} \phi_{i}^{\prime}\left(v_{\mathrm{eq}}\right)}{\lambda+P_{i}\left(v_{\mathrm{eq}}\right)}\right)
$$

and $P_{i}(v)=\phi_{i}(v)-r_{i}$.

Let us show that $\operatorname{Re} \lambda<0$. In fact, taking the complex conjugate to (17) and summing these equations, we have

$$
\operatorname{Re} \lambda+D+\operatorname{Re} G=0,
$$

where

$\operatorname{Re} G=\sum_{i \in \mathbf{I}_{\text {eq }}} c_{i}\left(x_{i} \phi_{i}^{\prime}\left(v_{\mathrm{eq}}\right)+\phi_{i}\left(v_{\mathrm{eq}}\right) \frac{x_{i} \phi_{i}^{\prime}\left(v_{\mathrm{eq}}\right)\left[\operatorname{Re} \lambda+P_{i}\left(v_{\mathrm{eq}}\right)\right]}{\left|\lambda+P_{i}\left(v_{\mathrm{eq}}\right)\right|^{2}}\right)$.

This implies that

$$
\operatorname{Re} \lambda \leqslant-D-\sum_{i \in \mathbf{I}_{\mathrm{eq}}} c_{i} x_{i} \phi_{i}^{\prime}\left(v_{\mathrm{eq}}\right) \quad \text { or } \operatorname{Re} \lambda \leqslant-\min _{i \in \mathbf{I}_{\mathrm{eq}}} P_{i}\left(v_{\mathrm{eq}}\right) .
$$

Thus the equilibrium point $\left(x_{1}, \ldots, x_{M}, v_{\text {eq }}\right)$ is locally stable for all $D$.

\section{EXTINCTIONS}

System (1) and (2) does not take into account species extinctions due to extinction thresholds. Here we present a model describing this effect. The system thereby handles the evolution to the final set of species. We follow [21] with essential simplifications since we do not take into account the emergence of new species. We start from random values of the model parameters.

The main parameters of our model in this section are the coefficients $r_{i}, K_{i}, a_{i}$, and $\gamma_{i}$. Let us introduce the vector parameter $\mathbf{P}_{i}=\left(r_{i}, K_{i}, a_{i}, \gamma_{i}\right)$. Note that $c_{i}$ is a species-specific parameter not necessary to include in this analysis and assumed to be fixed.

Let $\mathbf{P}=\left(P_{1}, P_{2}, P_{3}, P_{4}\right)$ be a random vector with a probability density function $\xi(\mathbf{P})$. This means that the values $\mathbf{P}_{i}$ are defined by random sampling, i.e., the parameters of the species are random independent vectors $\mathbf{P}_{i}$ that are drawn from the cone $\mathbf{R}_{+}^{4}=\left\{\mathbf{P}: P_{1}>0, P_{2}>0, P_{3}>0, P_{4}>0\right\}$ by the density $\xi$. Our assumption to $\xi$ can be formulated as follows:

Assumption I. The probability density function $\xi$ is a continuous function with a support, which has a compact closure in the positive cone $\mathbf{R}_{+}^{4}$.

The function $\xi$ is positive on $S_{\xi}$, where $S_{\xi}$ is an open and bounded set. The closure of $S_{\xi}$ we denote by $\bar{S}_{\xi}$. Assumption I implies that the mortality rates do not approach zero, and resource consumption is restricted. It is supposed that initial 
data $\bar{x}_{i}=x_{i}(0)$ are random mutually independent numbers drawn according to a density distribution

$$
\bar{x}_{i} \in \mathscr{X}\left(\bar{X}, \sigma_{X}\right)
$$

with the mean $\bar{X}$ and the deviation $\sigma_{X}$. The random assembly of the species defines an initial state of the ecosystem for $t=0$.

To describe species extinction, we introduce a small positive parameter $X_{\text {ext }}$ as an extinction threshold. We represent the set of indices $I_{M}=\{1,2, \ldots, M\}$ as a union of the two disjoint sets:

$$
I_{M}=S_{e}(t) \cup S_{v}(t), \quad t \geqslant 0 .
$$

Here $S_{e}(t)$ is the set of indices of species that exist at the time $t$, and $S_{v}(t)$ is the set of indices of species that have disappeared by the moment $t$. Let $N(t)$ denote the number of species in $S_{v}(t)$ at the moment $t, N(0)=M$. We assume that $S_{e}(0)=$ $\{1,2, \ldots, M\}$ and $S_{v}(0)=\emptyset$. In our model, the species with abundance $x_{k}$ vanishes at the moment $t_{*}$ if $x_{k}\left(t_{*}\right)=X_{\text {ext }}$ and $x_{k}(t)>X_{\text {ext }}$ for $t<t_{*}$. The parameter $X_{\text {ext }}$ can be interpreted as a threshold for species abundances.

The time evolution of the sets $S_{e}(t)$ and $S_{v}(t)$ can be described as follows:

(a) If the $k$ th species vanishes at a certain moment $t_{*}$, i.e., $k \in S_{e}(t)$ for $t<t_{*}$ and $x_{k}\left(t_{*}\right)=X_{\text {ext }}$, then the index $k$ moves from $S_{e}(t)$ to $S_{v}(t)$ at this moment $t=t_{*}$ and we set $x_{k}(t)=0$ for all $t>t_{*}$.

(b) We assume that the evolution stops at the moment $t_{\text {end }}$ if at this moment $S_{e}(t)=\emptyset$.

With the modifications described above, Eqs. (1) and (2) define the dynamics as follows. Within each time interval $\left(t_{*}, T_{*}\right)$ between the subsequent species extinctions, the dynamical evolution of $x_{i}(t)$ is defined by the system (1) and (2).

The quantity $N(t)$ is a piecewise constant decreasing function, therefore there exists a limit

$$
N(t) \rightarrow N_{f}, \quad t \rightarrow+\infty,
$$

where $N_{f}$ is the number of species that survived to the limit state (note that it is possible that $N_{f}=0$ ).

Let us introduce the parameters

$$
\delta_{i}=X_{\mathrm{ext}} \gamma_{i} / a_{i}
$$

and assume that

$$
\Phi\left(S_{0}, K_{i}\right)>\rho_{i}=p_{i}+\delta_{i}
$$

for some $i$. Condition (22) means that the resource supply is large enough for the existence of a positive equilibrium.

\section{DYNAMICS OF THE MODEL WITH EXTINCTIONS}

From (20) there exists a time moment $T_{f}$ such that all extinctions have occurred and thus we can use Theorem I and its corollary for the remaining species. According to Sec. IV, $S_{e}\left(T_{f}\right)$ is the set of indices corresponding to the species that exist for all $t>0$. That set contains $N_{f}=N\left(T_{f}\right)$ indices. We modify Eq. (9) as follows:

$$
D\left(S_{0}-v_{\text {eq }}\right)=F_{\text {ext }}\left(v_{\text {eq }}, b, K, p\right),
$$

where

$$
F_{\text {ext }}(v, b, K, p)=\sum_{i \in S_{e}\left(T_{f}\right)} R_{i}(v, b, K, p) .
$$

Corollary II. For sufficiently large $D>0$ or $\gamma_{0}>0$, all the solutions $(x(t), v(t))$ of (1) and (2) with positive initial data converge, as $t \rightarrow \infty$, to an equilibrium point defined by Eqs. (10), (23), and (24). That equilibrium depends on the set of remaining species $S_{e}\left(T_{f}\right)$.

The assertion follows from the arguments at the beginning of this section and Corollary I.

Note that the set $S_{e}\left(T_{f}\right)$ depends on initial data, therefore, in contrast to Theorem I, we have a number of possible final equilibria. To show this, let us consider the following situations. Let $M=3$, and for $X_{\text {ext }}$ all three species survive, thus $N_{\text {eq }}=3$.

Let $X_{\text {ext }}>0$ and $x_{3}(0)=X_{\text {ext }}+\kappa$, where $\kappa>0$ is a small number. We assume that $x_{1}(0)-X_{\text {ext }}$ and $x_{2}(0)-X_{\text {ext }}$ are not small. Suppose, moreover, that $D\left[S_{0}-v(0)\right]-F_{M}[v(0)]<0$ and $\left|D\left[S_{0}-v(0)\right]-F_{M}[v(0)]\right|>>\kappa$. Then it is clear that $x_{3}$ will become extinct within a short time period, and thus the third species is not involved in the set $S_{e}\left(T_{f}\right)$ of final equilibria. If $\kappa$ is not small, then the set contains the third species.

\section{CONCENTRATION OF SPECIES TRAITS}

Let us consider the case of arbitrary parameter values, supposing that the initial number of species $M \gg 1$. For each $\epsilon>0$, let us denote by $W_{\epsilon}(z)$ the set of the points in $\bar{S}_{\xi}$ that lie in the ball of radius $\epsilon$ centered at $z=(r, K, a, \gamma)$. The $\epsilon$-neighborhood $W_{\epsilon}(B)$ of a subset $B \subset \bar{S}_{\xi}$ is the union of $\epsilon$-neighborhoods $W_{\epsilon}(z)$ taken over all the points $z \in B$.

In the set $\bar{S}_{\xi}$ we introduce the partial order $\leqslant_{e}$ : $\left(r_{i}, K_{i}, a_{i}, \gamma_{i}\right) \leqslant_{e}\left(r_{j}, K_{j}, a_{j}, \gamma_{j}\right)$ if $a_{i} \leqslant a_{j}, r_{i} \geqslant r_{j}, K_{i} \geqslant K_{j}$, and $\gamma_{i} \geqslant \gamma_{j}$.

Consider the points $z_{*}=\left(r_{*}, K_{*}, a_{*}, \gamma_{*}\right)$, which are maximal with respect to the order $>_{e}$ in the set $\bar{S}_{\xi}$. Since that set is closed, bounded from below with respect to $K, r, \gamma$, and bounded from above with respect to $a$, the set $B_{*}$ of the points $z_{*}$ is not empty. It is clear that $B_{*}$ is a subset of the boundary $\partial S_{\xi}$.

Theorem III (concentration of traits). Let assumption I and (22) hold and let $\epsilon>0$ be a number. Then the parameters $a_{i}$, $r_{i}, \gamma_{i}$, and $K_{i}$ of species $x_{i}$ such that $x_{i}(t)>X_{\text {ext }}$ for all $t>0$ lie in the domain $W_{\epsilon}\left(B_{*}\right)$ with the probability $\operatorname{Pr}_{M}(\epsilon)$ such that $\operatorname{Pr}_{M}(\epsilon) \rightarrow 1$ as $M \rightarrow+\infty$.

The proof can be found in the Appendix.

If the set $B_{*}$ is a singleton (i.e., it consists of a single point), then we have the concentration trait effect, i.e., all essential parameters of the ecosystem become almost identical as a result of extinctions. Note that the set $B_{*}$ is a singleton in the case when $S_{\xi}$ is a box, i.e.,

$$
\begin{aligned}
S_{\xi}= & \left\{a_{-}<a_{i}<a_{+}, r_{-}<r_{i}<r_{+},\right. \\
& \left.K_{-}<K_{i}<K_{+}, \gamma_{-}<\gamma_{i}<\gamma_{+}\right\} .
\end{aligned}
$$

The set $B_{*}$ can have a more complicated structure, e.g., it may be a union of isolated points or a curve. Also note that even in the singleton case, species may differ in coefficients $c_{i}$.

\section{LIMITS OF BIODIVERSITY IN A STRESS ENVIRONMENT}

The following assertion gives us information on the limits of biodiversity for arbitrary parameter values, and our results 
are valid for arbitrary system dynamics: we do not use any assumptions here on the existence of globally attracting equilibria. Recall that $N_{f}$ is the number of species that survive as $t \rightarrow+\infty$, i.e., the corresponding abundances $x_{i}(t) \geqslant X_{\text {ext }}$ for all $t \geqslant 0$.

Proposition II. The number $N_{f}$ is bounded by a constant independent of $M$, namely

$$
N_{f}<N_{\max }=\left[\frac{D S_{0}}{X_{\text {ext }} a_{0} c_{0}\left(p_{0}+\delta_{0}\right)}\right]+1,
$$

where $[x]$ denotes the integer part of $x, c_{0}=\min c_{i}$, and

$$
a_{0}=\min _{r, a, K, \gamma \in \bar{S}_{\xi}} a, \quad \delta_{0}=\min _{r, a, K, \gamma \in \bar{S}_{\xi}} \delta, \quad p_{0}=\min _{r, a, K, \gamma \in \bar{S}_{\xi}} p .
$$

Proof. First we use an idea from [22]. Let $\langle F\rangle_{T}=$ $T^{-1} \int_{0}^{T} F(s) d s$ be the average of a function $F$ on $[0, T]$. The average of $F$ on $[0,+\infty)$ we denote by $\langle F\rangle$. By averaging (2), one obtains

$$
T^{-1}[v(T)-v(0)]=D\left(S_{0}-\langle v\rangle_{T}\right)-\sum_{i=1}^{M} c_{i} a_{i}\left\langle x_{i} \Phi\left(v, K_{i}\right)\right\rangle_{T} .
$$

Since the left-hand side here tends to 0 as $T \rightarrow+\infty$, Eq. (26) leads to

$$
D\left(S_{0}-\langle v\rangle\right)=\sum_{i=1}^{M} c_{i} a_{i}\left\langle x_{i} \Phi\left(v, K_{i}\right)\right\rangle,
$$

which in turn entails the estimate

$$
N_{f} a_{0} c_{0} X_{\mathrm{ext}}\left\langle\Phi\left(v, K_{*}\right)\right\rangle<D S_{0},
$$

where $K_{*}=\max _{i \in S_{e}\left(T_{f}\right)} K_{i}$. Consider the equation in (1) with the index $i \in S_{e}\left(T_{f}\right)$ for which $K_{i}=K_{*}$. Dividing both sides there by $x_{i}$, averaging, and using the fact that $x_{i}$ is bounded and separated from zero by $X_{\text {ext }}$, we get

$$
\left\langle\phi_{i}(v)\right\rangle-r_{i}=\gamma_{i}\left\langle x_{i}\right\rangle \geqslant \gamma_{i} X_{\mathrm{ext}} .
$$

Hence

$$
\left\langle\Phi\left(v, K_{*}\right)\right\rangle \geqslant p_{0}+\delta_{0} .
$$

This together with (28) leads to (25).

To find more precise estimates, we assume that coefficients $c_{i}, a_{i}, \gamma_{i}$, and $r_{i}$ satisfy

$$
\begin{aligned}
& C_{-} a<a_{i}<C_{+} a, \quad C_{-} c<c_{i}<C_{+} c, \quad 1 \leqslant i \leqslant M, \\
& C_{-} \gamma<\gamma_{i}<C_{+} \gamma, \quad C_{-} r<r_{i}<C_{+} r, \quad 1 \leqslant i \leqslant M,
\end{aligned}
$$

where $a, c, \gamma$, and $r$ are characteristic values of the corresponding coefficients, and $C_{ \pm}$are positive constants independent of $M, a, c, \gamma$, and $r$. Let us introduce the stress parameter by

$$
P_{\text {stress }}=\frac{c a^{2}}{\gamma D S_{0}} \text {. }
$$

To simplify the statement, we also suppose that $K_{i}=$ $K$. The general assertion on the trait concentration can be formulated as follows:

Proposition III. Suppose Assumption I and condition (22) hold. Let $i, j$ be two indices such that the corresponding species abundances $x_{i}(t), x_{j}(t)$ satisfy $x_{i}(t)>X_{\mathrm{ext}}, x_{j}(t)>X_{\mathrm{ext}}$ for all $t \geqslant 0$. Then

$$
\left|p_{j}-p_{i}\right|<C_{0} P_{\text {stress }}^{-1}\left(p_{0}+\delta_{0}\right)^{-1},
$$

where $C_{0}>0$ does not depend on $a, c, \gamma, r$, and $X_{\text {ext }}$.

Proof. Consider the species such that $x_{i}(t)>X_{\text {ext }}$ for all $t \geqslant$ 0 . The corresponding set of indices we denote by $S_{e}$. Averaging Eqs. (1) for the species $x_{i}$ with $i \in S_{e}$, we obtain the following relation:

$$
\gamma_{i}\left\langle x_{i}^{2}\right\rangle=a_{i}\left\langle x_{i}\left[\Phi(v, K)-p_{i}\right]\right\rangle .
$$

Furthermore, we divide (1) on $x_{i}$ and average the obtained equation that gives

$$
\left\langle x_{i}\right\rangle=a_{i} \gamma_{i}^{-1}\left\langle\Phi(v, K)-p_{i}\right\rangle .
$$

The Cauchy inequality implies $\left\langle x_{i}^{2}\right\rangle \geqslant\left\langle x_{i}\right\rangle^{2}$. Therefore, (34) and (35) entail

$$
\left\langle x_{i}\left(\Phi-p_{i}\right)\right\rangle \geqslant a_{i} \gamma_{i}^{-1}\left\langle\Phi-p_{i}\right\rangle^{2},
$$

where, for brevity, we use the notation $\Phi=\Phi(v, K)$. From (2) we have

$$
D S_{0} \geqslant \sum_{i \in S_{e}} c_{i} a_{i}\left\langle x_{i} \Phi\right\rangle
$$

We observe that

$$
\left\langle x_{i} \Phi\right\rangle=\left\langle x_{i} \Phi-x_{i} p_{i}+x_{i} p_{i}\right\rangle .
$$

From the above identity and (34), one has

$$
\left\langle x_{i} \Phi\right\rangle=\left\langle x_{i}\left(\Phi-p_{i}\right)\right\rangle+a_{i} \gamma_{i}^{-1} p_{i}\left\langle\Phi-p_{i}\right\rangle .
$$

The above relation and (36) and (37) lead to the inequality

$$
D S_{0} \geqslant\langle\Phi\rangle \sum_{i \in S_{e}} c_{i} a_{i}^{2} \gamma_{i}^{-1}\left\langle\Phi-p_{i}\right\rangle,
$$

which, with (29), can be rewritten as follows:

$$
P_{\text {stress }}^{-1} \geqslant\langle\Phi\rangle \sum_{i \in S_{e}} \beta_{i}\left\langle\Phi-p_{i}\right\rangle
$$

where $\beta_{i}=c_{i} a_{i} \gamma_{i}(c a \gamma)^{-1}$ are bounded coefficients independent of $a, \gamma$, and $c$. Estimate (39) entails

$$
\left[\Phi(v, K)-p_{l}\right]_{+}<C_{2} P_{\text {stress }}^{-1}\langle\Phi\rangle^{-1} \quad \forall l \in S_{e}
$$

for some $C_{2}>0$, which is independent of $\gamma, a, c$, and $r$. From (35), $\Phi-p_{i}$ is positive and hence the index + in (40) can be removed. Combining (40) for $l=i$ and $j$ and taking into account that $\langle\Phi\rangle>p_{0}+\delta_{0}$, one has (33).

Let us derive an estimate of $N_{f}$ via $P_{\text {stress }}>>1$ and the average $\langle\Phi\rangle$. We suppose that $p_{i}=p_{0}+(i-1) \Delta p, \Delta p \ll 1$, and $\beta_{i}=\beta=O(1)$. Then estimate (39) implies

$$
N_{f} \leqslant 2 \beta^{-1} P_{\text {stress }}^{-1}\left[\left(\langle\Phi\rangle-p_{0}\right)\langle\Phi\rangle\right]^{-1} \text {. }
$$

This calculation is consistent with numerical simulations. For $\gamma=0.00001$, when all other parameters have the order 1, we obtain a strong concentration effect (see Fig. 1). Computations were done for a population of $M=50$ species, where random parameters are chosen as explained above.

As a measure of the trait concentration, we can use the quantity

$$
\operatorname{var}(p)=\max _{i}\left\{p_{i}\right\}-\min _{i}\left\{p_{i}\right\}
$$




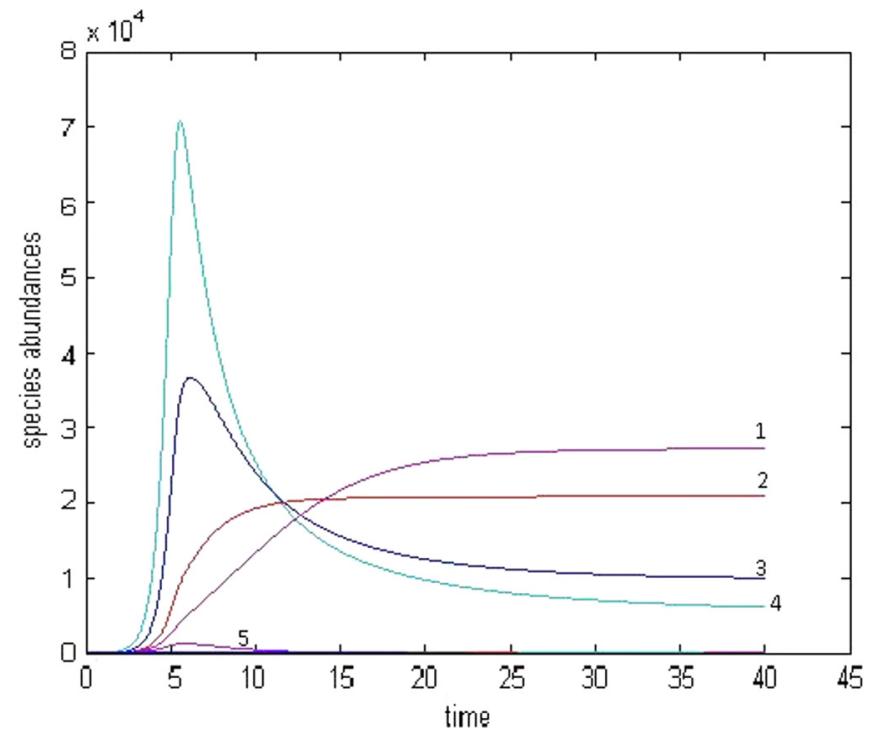

FIG. 1. Dynamics of a large population with a very small $\gamma$. The graphs of the species abundances are $x_{i}(t)$, and the species number is $M=50$. The parameters are as follows: $K=4, D=10, S=100$, $E_{y}=1, s_{y}=0.3, E_{a}=2, s_{a}=0.2, E_{r}=1-\ln (20), s_{r}=0.1$, and $\gamma=10^{-5}$. Here four species coexist instead of a single one (they are indicated by numbers $1-4)$.

Then the initial $\operatorname{var}(p) \approx 0.6$, but for four remaining species with large abundances we have $\operatorname{var}(p)=0.07$. We see that these four species are abundant whereas all other species are extremely rare. Note that these asymptotic results can be generalized in the case of different $K_{i}$.

\section{A. Mass extinctions: An analytical approach}

Relation (25) allows us to describe, in an analytical way, mass extinctions. Mass extinctions may result as a consequence of a sharp change of some environmental parameter. It is natural to assume that climate variations or other abiotic ones can reduce the resource supply level $S_{0}$. Assume, for example, that this reduction is $\Delta S_{0}>0$ and the new resource supply $S_{\text {new }}=S_{0}-\Delta S_{0}$ satisfies

$$
S_{\text {new }}<X_{\text {ext }} a_{0} c_{0}\left(p_{0}+\delta_{0}\right) .
$$

The preceding equation implies that for sufficiently large $\Delta S_{0}$, even all species may become extinct. We therefore refer to this level of the resource $S_{\text {new }}$ as a catastrophic level.

Note that these analytical results show that there are interesting phenomena. First, let us compare two ecosystems. One is a random assembly of many species where the variation $\operatorname{var}(p)$ defined by (42) is large, and the other ecosystem is a result of a long evolution leading to the concentration, i.e., $\operatorname{var}(p)$ is small. We find that the concentrated system is more stable with respect to variations in the resource. Namely, a sharp change of $S_{0}$ will kill many more species in the first ecosystem than in the second one. Secondly, assume that catastrophes do occur several times yet with a fairly long time in between. Each catastrophe will reduce the biodiversity yet with less and less probability since the concentration effect becomes stronger and stronger.

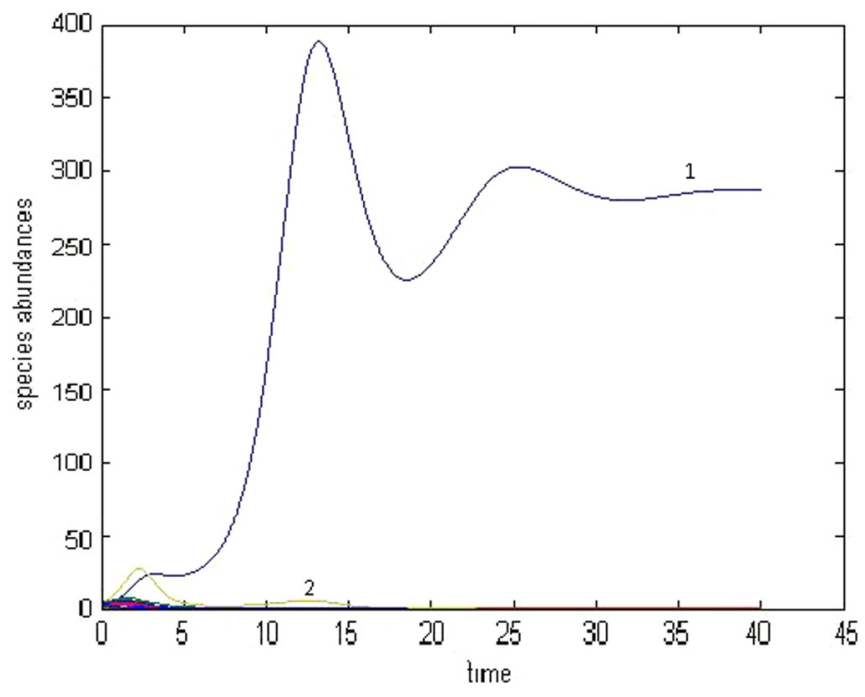

FIG. 2. Dynamics of a species community. The species number $M=50, D=0.1$, and $S_{0}=30$. We observe oscillations and finally that the competition exclusion principle works: only a single set of parameters remains. This can imply a single species (indicated by 1), especially if no other traits are important, as assumed in the model.

To investigate more realistic situations when $K_{i}$ are different and the parameters are random, we performed numerical simulations described in the following section.

\section{NUMERICAL SIMULATIONS}

In numerical simulations, the parameters are chosen as follows. The coefficients $a_{i}$ are independent and identically distributed (i.i.d.) random quantities such that each $\ln a_{i}$ is a normally distributed number with the mean $E_{a}=1$ and the standard deviation $\sigma_{a}=0.2$. This means that each $a_{i}$ has the same log-normal distribution, $a_{i} \in \ln \mathbf{N}\left(E_{a}, s_{a}\right)$.

Similarly, the coefficients $r_{i}$ are i.i.d. random quantities, $\ln r_{i}$ is normally distributed on $[0,1]$ number with the mean $E_{r}=0.1$, and the standard deviation $\sigma_{r}=0.03-\ln (20)$. The parameters $D=0.1, K=4, S=30$, and $\gamma_{i}=\gamma=0.001$. The coefficients $c_{i}$ are random numbers uniformly distributed on $[0,1]$ and normalized in such a way that $\sum c_{i}=1$. The initial data $X_{i}$ are i.i.d. random numbers distributed log-normally, $X_{i}=\exp \left(y_{i}\right), y_{i} \in \mathbf{N}\left(E_{y}, s_{y}\right)$, with parameters $E_{y}=1$ and $s_{y}=0.3$. We suppose that all $c_{k}, r_{j}$, and $a_{l}$ are mutually independent.

For these random species communities and $N=50$ we observe oscillations and then a convergence to an equilibrium (see Fig. 2).

Using simulations, we have considered the dependence of biodiversity and the concentration trait effect on the stress parameter for populations with random parameters $K_{i}, p_{i}$. The results are consistent with analytic considerations of the previous section, and they can be seen in Fig. 3.

In the numerical simulations of biodiversity, we can also observe the trait concentration. For the example illustrated by Fig. 3, the variation $\operatorname{var}(p)$ decreases very strongly as a result of species extinctions, and this reduction increases as the stress parameter increases. 


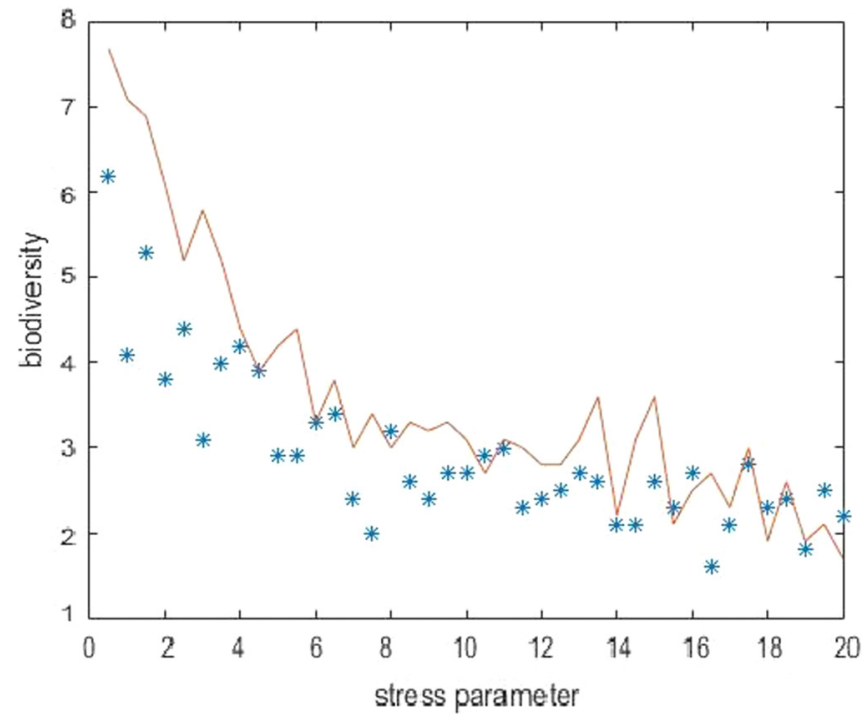

FIG. 3. The species number $M=100$. The species parameters $K$ and $p$ are random numbers obtained by log-normal distributions, $K=$ $\exp (\tilde{K}), p=\exp (\tilde{p})$, where $\tilde{K} \in \mathscr{N}\left(K_{0}, \sigma_{K}\right)$ and $\tilde{K} p \in \mathscr{N}\left(p_{0}, \sigma_{p}\right)$. The star curve corresponds to the case $K_{0}=1, \sigma_{K}=0, p_{0}=-1$, and $\sigma_{p}=0.2$. For the continuous curve the parameters are the same, but we have a variation in $K: \sigma_{K}=0.5$.

\section{DYNAMICS OF THE LIMIT ECOSYSTEM}

According to Theorem III, if the set $B^{*}$ consists of a single point, for $M \gg 1$ the limit system (that appears as a result of many extinctions) has the property $p_{i} \approx p, K_{i} \approx K$, where $p, K$ are some parameter values. To understand the dynamics of that system, we consider system (1) and (2) for the case $p_{i}=$ $p$ and $K_{i}=K$. Let us introduce a new variable $Q=-p t+$ $\int_{0}^{t} \Phi(v(s), K) d s$. The variable $Q$ is an analog of "quality of life" introduced in [2] for the linear case $\Phi(v, K)=v$. This case is studied in [23]. The results of [23] can be extended to our limit model. We seek solutions to Eqs. (1) in the form

$$
x_{i}(t)=C_{i}(t) \exp \left[a_{i} Q(t)\right]
$$

where $C_{i}$ are new unknowns. From (1) one obtains

$$
\frac{d C_{i}}{d t}=-\gamma_{i} C_{i}^{2} \exp \left[a_{i} Q(t)\right], \quad C_{i}(0)=\bar{x}_{i},
$$

which gives

$$
\frac{d C_{i}}{d t}=-\gamma_{i} C_{i}^{2} \exp \left[a_{i} Q(t)\right] .
$$

By solving these equations, we find

$$
C_{i}=\frac{C_{i}(0)}{1+\gamma_{i} C_{i}(0) \int_{0}^{t} \exp \left[a_{j} Q\left(t^{\prime}\right)\right] d t^{\prime}} .
$$

Using the last relation, from (2) one obtains

$$
v=\frac{K P}{1-P}, \quad \frac{d v}{d t}=\frac{K}{(1-P)^{2}} \frac{d P}{d t},
$$

where $P=d Q / d t+p$.
After some straightforward computations, Eqs. (1) and (2) reduce to the system

$$
\begin{gathered}
\frac{K}{(1-P)^{2}} \frac{d P}{d t}=D\left(S_{0}-\frac{K P}{1-P}\right)-P f(Q(\cdot)), \\
\frac{d Q}{d t}=P-p,
\end{gathered}
$$

where

$$
f(Q(\cdot))=\sum_{j=1}^{N} \frac{c_{j} a_{j} \bar{x}_{j} \exp \left[a_{j} Q(t)\right]}{1+\gamma_{j} \bar{x}_{j} \int_{0}^{t} \exp \left[a_{j} Q\left(t^{\prime}\right)\right] d t^{\prime}} .
$$

Equation (45) describes a nonlinear oscillator with a damping term and nonlinearities with a time delay. Note that $f$ depends on initial data $\bar{x}_{i}$. So, we see that the limit ecosystem can be considered as a nonlinear oscillator with a friction and a memory. The oscillator state is determined by two variables: $P$ and $Q$. The first variable is a difference between the normalized species consuming rate $\Phi(v, K)$ and the normalized species mortality rate, i.e., it allows for a biological interpretation. This variable can be called the Malthusian parameter. The variable $Q$ does not allow for a simple explicit interpretation. It is a generalization of the quality of life introduced by Volterra. Note that $Q$ is the integral of $P$, i.e., it can be considered as an integral Malthusian parameter. Since this is a parameter expressing a trait over a long period of time, we can call $P$ the sustainable Malthusian parameter.

Equation (45) can be simplified in two cases: for $\gamma=0$ and for bounded times, $t \ll \ln \left(\gamma^{-1}\right)$ (an initial stage) and $t \gg 1 / \gamma$ (large times, the final stage). In the first case from (47) we have

$$
f(Q(\cdot))=f(Q(t))=\sum_{j=1}^{N} c_{j} a_{j} \bar{x}_{j} \exp \left[a_{j} Q(t)\right] .
$$

We obtain an oscillator, which is a perturbed Hamiltonian integrable system without memory. In this case, for small $D$ the solutions of (45) tend to equilibrium in an oscillating manner (see Fig. 2).

\section{CONCLUSION}

In this paper, we have investigated a model of ecosystems exploiting a single resource and interacting with the environment. Until May's seminal works [24,25], ecologists believed that large complex ecosystems, involving a larger number of species and interconnections, are stable. May [24,25] considered a community of $S$ species with connectance $C$ that measures the number of realized links with respect to the number of all possible links. May's analysis of the local stability of an equilibrium produced results that were quite revolutionary and inspired a great deal of discussion. It was shown that for large systems with random interaction parameters, instability can occur for large $C$. Communities that are more connected are more unstable. This approach was developed in [19,26], which studied more complicated networks with interactions of different types (predator-prey, amensalism, mutualism, and competition).

All of these fundamental results hold under the assumption that, at an equilibrium, ecosystems have a random structure. In other words, the entries of the matrix, which define 
the linearization of a system at equilibrium, are distributed according to smooth densities, e.g., Gaussian densities.

In this paper, we use a similar assumption but on the initial choice of species traits. The initial distribution of species traits is defined by continuous densities with nonempty supports, i.e., roughly speaking, the species traits are distributed homogeneously in a domain. We show that in the evolution process, the distribution of species traits becomes more concentrated when an ecosystem evolves under a stress or as a result of species extinctions. During the evolution process, the domain of species trait localization shrinks. That small domain of localization means that species become increasingly similar (as in [18]). In contrast to [18], we do not use any specific assumptions on the adaptation of system parameters. We have found a parameter that defines the stress level. This parameter depends on the supply level, turnover rate, and resource-consuming intensity.

The most interesting effect of species trait concentration is as follows. For large times, a stable and simple limit ecosystem appears just because most species become extinct under stress. For large times, ecosystem dynamics and extinctions of species under stress produce a self-organized community consisting of species with close consumer efficiencies (note that these species can be different in other traits). In some cases, the dynamics of this limit community can be described by a simple equation that describes a nonlinear oscillator with a friction and a memory, which is close to a Hamiltonian system. We have found an asymptotic approach to study this system. Note that the reduction mechanism differs from the one previously found in $[1,23]$. In [1], a mean-field approach is applied to complex ecosystems and gene networks. This approach exploits the system topology when species (genes) can interact with many others. Complicated systems of equations were reduced to a single differential equation of first order. Such equations do not exhibit time oscillations, whereas our equation simulates a perturbed nonlinear oscillator, and it can describe slowly decreasing oscillations. In [23], a reduction to Hamiltonian systems is also based on the topological properties of interactions in ecosystems. So, the reduced descriptions of complex systems proposed in $[1,23]$ can be called topological. In contrast to $[1,23]$, in the present paper the reduced description is based not only on the system topology (i.e., the fact that species share the same resource) but also on other phenomena, namely extinctions and selection by a tough environment (which can be measured by the stress parameter).

These results can be useful for understanding why ecosystems where species feed on few resources can have a large biodiversity, and how mass extinctions depend on environment and ecosystem parameters. The intriguing effect is that we observe a picture similar to statistical physics: the state of an ecosystem that arises as a product of a long evolution can be described by two quantities, $P, Q$, which have a biological interpretation. Namely, $P$ can be called the Malthusian parameter, and this quantity determines a balance between mean mortality and growth rates. The quantity $Q$ can be named the sustainable Malthusian parameter, and it can be obtained by integrating $P$ over time.

We have computed analytically the number of finally coexisting and surviving species, and how this number depends on the main parameters of the ecosystem (i.e., the resource supply, the mortality rates, the resource turnover, etc.). It is shown that the main quantity that determines the final biodiversity is the stress parameter.

\section{ACKNOWLEDGMENTS}

The authors are thankful to the referee for useful remarks. S.V. was supported by Linkoping University and by the Government of the Russian Federation through Mega-grant No. 074-U01 and RFBR Grant No. 16-01-00648.

\section{APPENDIX}

\section{Proof of formula (17)}

Assume that all $P_{i}\left(v_{\mathrm{eq}}\right)>0$. Then the eigenvalue problem for the linear part of the right-hand side of (1) and (2) at the equilibrium point $\left(x_{1}, \ldots, x_{M}, v_{\mathrm{eq}}\right)$ has the form

$$
\begin{gathered}
-\gamma_{i} x_{i} X_{i}+x_{i} \phi_{i}^{\prime}\left(v_{\mathrm{eq}}\right) V=\lambda X_{i}, \quad i=1, \ldots, M, \\
-\sum_{i=1}^{M} c_{i} \phi_{i}\left(v_{\mathrm{eq}}\right) X_{i}-\left(D+\sum_{i=1}^{M} c_{i} x_{i} \phi_{i}^{\prime}\left(v_{\mathrm{eq}}\right)\right) V=\lambda V
\end{gathered}
$$

where $\left(X_{1}, \ldots, X_{M}, V\right)$ is an eigenvector corresponding to the eigenvalue $\lambda$. Solving the first system with respect to $X_{i}$ and inserting the solution into the second equation, we obtain

$$
X_{i}=\frac{x_{i} \phi_{i}^{\prime}\left(v_{\mathrm{eq}}\right)}{\lambda+\gamma_{i} x_{i}}
$$

and

$$
\lambda+D+\sum_{i=1}^{M} c_{i} x_{i} \phi_{i}^{\prime}\left(v_{\mathrm{eq}}\right)+\sum_{i=1}^{M} c_{i} \phi_{i}\left(v_{\mathrm{eq}}\right) \frac{x_{i} \phi_{i}^{\prime}\left(v_{\mathrm{eq}}\right)}{\lambda+\gamma_{i} x_{i}}=0
$$

Since $\gamma_{i} x_{i}=P_{i}\left(v_{\mathrm{eq}}\right)$ at the equilibrium point, we arrive at (17).

If some of $P_{i}\left(v_{\text {eq }}\right)$ are nonpositive, the corresponding terms in (A1)-(A3) are zeros and we again arrive at (17).

\section{Proof of Theorem I on the global stability of positive solutions}

We apply a special method based on the theory of decreasing operators in Banach spaces (see [27] and references therein) that allows us to prove this assertion without any additional assumptions. This approach is applicable here due to the special properties of monotonicity in our problem.

Let us rewrite (12) as follows:

$$
\bar{v}=V(\bar{v}),
$$

where the operator $V$ is described in Sec. III A. We remind the reader that $V(\bar{v})$ is a decreasing function in $\bar{v}$.

Our next step is to rewrite system (1) and (2) as an integral equation for an unknown function $v(t)$. Let $w(t)$ be a given non-negative, continuous, bounded function on $[0, \infty)$ having a limit $\bar{w}$ at infinity. We can resolve Eqs. (1) (with $v$ replaced by $w$ ) following Sec. IX. As a result, we obtain

$$
x_{i}(t)=\mathbf{X}_{i}(w(\cdot))(t)
$$


where

$$
\mathbf{X}_{i}(w(\cdot))(t)=\frac{x_{i}(0)}{J_{i}(w(\cdot))(t)}
$$

and

$$
\begin{aligned}
J_{i}= & \exp \left(-\int_{0}^{t} P_{i}(w(s)) d s\right) \\
& +\gamma_{i} x_{i}(0) \int_{0}^{t} \exp \left(-\int_{t_{1}}^{t} P_{i}(w(s)) d s\right) d t_{1} .
\end{aligned}
$$

One can verify that for $x_{i}(0)>0$,

$$
\mathbf{X}_{i}(w)(t) \rightarrow X_{i}(\bar{w}) \quad \text { as } t \rightarrow \infty,
$$

where $X_{i}$ is defined in Sec. III A.

Next, we can solve Eq. (2) with respect to $v$, where $x_{i}$ is given by (A5) and $v(0)=v_{0}$. We denote this solution by $\mathbf{V}(t)=\mathbf{V}(w(\cdot))(t)$. We cannot write this solution explicitly, but in what follows we need only some of its properties. First, this solution is a decreasing function with respect to $x_{i}$ and consequently with respect to $w$. Second,

$$
\mathbf{V}(w(\cdot))(t) \rightarrow \bar{v} \quad \text { as } t \rightarrow \infty,
$$

where $\bar{v}=V(\bar{w})$. Thus the unique solution to the problem (1) and (2) with the Cauchy data (4) can be obtained by solving the following fixed-point problem:

$$
v(t)=\mathbf{V}(v)(t)
$$

and then

$$
x_{i}(t)=\mathbf{X}_{i}(v(\cdot))(t), \quad i=1, \ldots, M .
$$

To solve the equation $v=\mathbf{V}(v(\cdot))$ in the class of bounded, continuous, non-negative functions (denoted by $\mathbf{B}$ ), we use the following iterations:

$$
v_{n+1}(t)=\mathbf{V}\left(v_{n}(\cdot)\right)(t), \quad n=1,2, \ldots, \quad v_{0}(t)=0 .
$$

Then

$$
\begin{aligned}
v_{0} \leqslant v_{2} & \leqslant v_{4} \leqslant \cdots, \quad v_{1} \geqslant v_{3} \geqslant \cdots, \quad \text { and } \\
v_{2 j} & \leqslant v_{2 k+1} \quad \text { for all } j, k
\end{aligned}
$$

(here $\leqslant$ denotes the partial order on $\mathbf{B}: v \leqslant u$ if $u(t) \leqslant$ $v(t) \forall t \in[0, T])$.

To show the convergence of the odd and even iterations, we observe that we can consider the fixed-point equation (A6) on a finite interval $(0, T)$. Now the operator $\mathbf{V}: C[0, T] \rightarrow C[0, T]$ is compact and hence the odd and even terms of sequences converge on $[0, T]$ for each $T$. We introduce their limits

$$
\check{V}(t)=\lim _{j \rightarrow \infty} v_{2 j}(t), \quad \hat{V}(t)=\lim _{k \rightarrow \infty} v_{2 k+1}(t) .
$$

Then $\mathbf{V}(\check{V})=\hat{V}$ and $\mathbf{V}(\hat{V})=\check{V}$. Let $\hat{x}_{i}$ be given by (A5) with $w=\hat{V}$, and let $\check{x}_{i}$ be given by (A5) with $w=\hat{V}$. Then the vector function $\left(\check{x}_{1}, \ldots, \check{x}_{M}, \hat{V}\right)$ satisfies the problem

$$
\begin{aligned}
& \frac{d \check{x}_{i}}{d t}=\check{x}_{i}\left[-r_{i}+\phi_{i}(\check{V})-\gamma_{i} \check{x}_{i}\right], \quad i=1, \ldots, M, \\
& \frac{d \hat{V}}{d t}=D\left(S_{0}-\hat{V}\right)-\sum_{i=1}^{M} c_{i} \check{x}_{i} \phi_{i}(\hat{V}),
\end{aligned}
$$

and the functions $\left(\hat{x}_{1}, \ldots, \hat{x}_{M}, \check{V}\right)$ are solutions of

$$
\begin{aligned}
& \frac{d \hat{x}_{i}}{d t}=\hat{x}_{i}\left(-r_{i}+\phi_{i}(\hat{V})-\gamma_{i} \hat{x}_{i}\right), \quad i=1, \ldots, M, \\
& \frac{d \check{V}}{d t}=D\left(S_{0}-\check{V}\right)-\sum_{i=1}^{M} c_{i} \hat{x}_{i} \phi_{i}(\check{V}) .
\end{aligned}
$$

Moreover, the last two systems have the same Cauchy data. Taking the differences, we obtain a homogeneous Cauchy problem for $\left(\hat{x}_{1}-\check{x}_{1}, \ldots, \hat{x}_{M}-\check{x}_{M}, \hat{V}-\check{V}\right)$, and by uniqueness for the Cauchy problem we obtain that $\check{V}=\hat{V}$.

Let us turn to the asymptotic behavior of the fixed-point solutions. Let $\bar{v}_{k}=\lim _{t \rightarrow \infty} v_{k}(t)$. Then

$$
\bar{v}_{0}=0 \quad \text { and } \bar{v}_{k+1}=V\left(\bar{v}_{k}\right), \quad k=0, \ldots .
$$

This proves inequalities (15) and (16) and completes the proof of Theorem I.

\section{Proof of Theorem III}

This proof proceeds in three steps.

Step 1: Monotonicity of species abundances. Consider a point $\bar{z}=\left(a_{i}, r_{i}, \gamma_{i}, K_{i}\right)$, which is not contained in $W_{\epsilon}\left(B_{*}\right)$, and the corresponding species population $x_{i}(t)$. Suppose that for all $t \geqslant 0$ we have

$$
x_{i}(t)>X_{\text {ext }}
$$

Consider the $j$ th species with parameters $\left(a_{j}, r_{j}, \gamma_{j}, K_{j}\right)$ and the species abundance $x_{j}(t)$. We assume that

$$
x_{j}(0) \geqslant x_{i}(0), \quad r_{i} \geqslant r_{j}, \quad a_{i} \leqslant a_{j}, \quad \gamma_{i} \geqslant \gamma_{j}, \quad K_{i} \geqslant K_{j} .
$$

Then

$$
x_{j}(t) \geqslant x_{i}(t) \quad \forall t>0 .
$$

Indeed, let us consider equations for $x_{i}, x_{j}$ :

$$
\begin{aligned}
\frac{d x_{i}}{d t} & =x_{i}\left[-r_{i}+\phi_{i}(v)-\gamma_{i} x_{i}\right], \\
\frac{d x_{j}}{d t} & =x_{j}\left[-r_{j}+\phi_{j}(v)-\gamma_{j} x_{j}\right] .
\end{aligned}
$$

If (A9) is violated, then there is a time moment $t_{1}>0$ such that

$$
x_{j}\left(t_{1}\right)=x_{i}\left(t_{1}\right), \quad \frac{d x_{i}}{d t}\left(t_{1}\right)>\frac{d x_{j}}{d t}\left(t_{1}\right) .
$$

But

$$
\begin{aligned}
& x_{i}\left(t_{1}\right)\left[-r_{i}+\phi_{i}(v)-\gamma_{i} x_{i}\left(t_{1}\right)\right] \\
& \quad \leqslant x_{i}\left(t_{1}\right)\left[-r_{i}+\phi_{i}(v)-\gamma_{i} x_{i}\left(t_{1}\right)\right]
\end{aligned}
$$

due to the first inequality in (A12) and (A8). The last inequality contradicts the second inequality in (A12), thus (A9) is proved.

Inequality (A9) shows that if the species $x_{i}$ survives for all times, then all the species with parameters satisfying (A8) also survive for all $t>0$.

Step 2: A priori boundedness of biodiversity. Here we use Proposition II. The number $N_{s}$ of species that survive for all 
times is a priori bounded by the system parameters and does not depend on $M$ as $M \rightarrow \infty$. We refer to the corresponding set of species parameters as $\mathscr{P}_{s}$. Due to Proposition II,

$$
N_{s}<C
$$

where $C>0$ is independent of $M$.

Step 3. Let us consider the $\epsilon$-neighborhood $W_{\epsilon}\left(B_{*}\right)$. Suppose there exists a point $\bar{z} \notin W_{\epsilon}\left(B_{*}\right)$. We denote the initial data $x_{i}(0)$ for the corresponding species by $\bar{x}_{i}$. Then, according to step 1 , the set $\mathscr{P}_{s}$ contains all points $z$ from $W_{\epsilon}\left(B_{*}\right)$ such that $z \geqslant_{e} \bar{z}$. We denote the set of such points by $W_{\epsilon, \bar{z}}\left(B_{*}\right)$. Note that due to the conditions of the set $S_{\xi}$ (see Assumption I), the set $W_{\epsilon, \bar{z}}\left(B_{*}\right)$ contains a small open ball. Therefore, since $\xi$ is positive on the interior of $S_{\xi}$ (see Assumption I), we have

$$
1>J=\int W_{\epsilon, \bar{z}}\left(B_{*}\right) \xi(z) d z>\delta_{\epsilon, \bar{z}}>0 .
$$

The number $\delta_{\epsilon, \bar{z}}$ is independent of $M$. Consider the event $E=A B$, where $A$ is the event in which the species parameters lie in $W_{\epsilon, \bar{z}}\left(B_{*}\right)$, and $B$ is the event in which the initial data $x_{i}(0)>\bar{x}_{i} \forall i$. The events $A$ and $B_{s}$ are independent, and $\operatorname{Prob}(A)>0$ due to the above estimate for $J$. According to the hypothesis on the random choice of $x_{i}(0)$, we also have $\operatorname{Prob}(B)>0$. Therefore, $\operatorname{Prob}(E)=q>0$.

Consider the event $E_{M, N_{s}}$ in which among $M$ species there are not more than $N_{S}$ species such that the corresponding species parameters lie in $W_{\epsilon, \bar{z}}\left(B_{*}\right)$ and the initial data $x_{i}(0)>$ $\bar{x}_{i} \forall i$. The probability of $E_{M, N_{s}}$ can be computed by the Bernoulli relation, and we have

$$
\operatorname{Prob}\left(E_{M, N_{s}}\right)<\sum_{k=0}^{N_{s}} M^{k}(k !)^{-1} q^{k}(1-q)^{M-k} .
$$

We see that $E_{M, N_{s}} \rightarrow 0$ as $M \rightarrow \infty$, and Theorem III is proved.
[1] J. Gao, B. Barzel, and A-L. Barabási, Nature (London) 530, 307 (2016).

[2] V. Volterra, Lecons sur la Theorie Mathematique de la Lutte pour la Vie (Gauthier-Villard, Paris, 1931).

[3] G. Hardin, Science 131, 1292 (1960).

[4] D. Tilman, Ecology 58, 338 (1977).

[5] E. C. Zeeman and M. L. Zeeman, Trans. Am. Math. Soc. 355, 713 (2003).

[6] G. E. Hutchinson, Am. Nat. 95, 137 (1961).

[7] S. Roy and J. Chattopadhyay, Ecol. Complex. 4, 26 (2007).

[8] J. D. Moll and J. S. Brown, Am. Natural. 171, 839 (2008).

[9] C. W. Harbison, Ecology 89, 3186 (2008).

[10] N. R. Record, A. J. Pershing, and F. Maps, ICES J. Mar. Sci. 71, 236 (2014).

[11] J. Huisman and F. J. Weissing, Nature (London) 402, 407 (1999).

[12] N. Loeuille and M. Loreau, Proc. Natl. Acad. Sci. (USA) 102, 5761 (2005).

[13] B. Drossel, A. J. McKane, and Ch. Quince, J. Theor. Biol. 229, 539 (2004).

[14] ̊̊. Brännström, N. Loeuille, M. Loreau, and U. Dieckmann, Theor. Ecol. 4, 467 (2011).
[15] A. J. McKane, Eur. Phys J. B 38, 287 (2004).

[16] M. Kondoh, Science 299, 1388 (2003).

[17] G. J. Ackland and I. D. Gallagher, Phys. Rev. Lett. 93, 158701 (2004).

[18] M. Scheffer and E. H. van Nes, Proc. Natl. Acad. Sci. (USA) 103, 6230 (2006).

[19] S. Allesina and Si. Tang, Nature (London) 483, 205 (2012).

[20] S. Allesina (private communication).

[21] V. Kozlov, S. Vakulenko, and U. Wennergren, Bull. Math. Biol. 78, 2186 (2016).

[22] J. Hofbauer and K. Sigmund, Evolutionary Games and Population Dynamics (Cambridge University Press, Cambridge, 1998).

[23] V. Kozlov, S. Vakulenko, and U. Wennergren, Phys. Rev. E 93, 032413 (2016).

[24] R. M. May, Nature (London) 238, 413 (1972).

[25] R. May, Stability and Complexity in Model Ecosystems (Princeton University Press, Princeton, NJ, 1974).

[26] S. Allesina, Nature (London) 487, 175 (2012).

[27] G. Herzog and P. C. Kunstmann, Numer. Funct. Anal. Optim. 34, 530 (2013). 